\title{
Management of pulmonary embolism: recent evidence and the new European guidelines
}

\author{
Stavros V. Konstantinides ${ }^{1,2}$ and Adam Torbicki ${ }^{3}$
}

\begin{abstract}
Affiliations: ${ }^{1}$ Center for Thrombosis and Hemostasis (CTH), University Medical Center Mainz, Mainz, Germany. ${ }^{2}$ Dept of Cardiology, Democritus University of Thrace, Alexandroupolis, Greece. ${ }^{3}$ Dept of Pulmonary Circulation and Thromboembolic Diseases, Center of Postgraduate Medical Education, ECZ-Otwock, Otwock, Poland.
\end{abstract}

Correspondence: Stavros V. Konstantinides, Center for Thrombosis and Hemostasis, University Medical Center Mainz, Langenbeckstrasse 1, Bldg. 403, 55131 Mainz, Germany.

E-mail: stavros.konstantinidesQunimedizin-mainz.de

@ERSpublications

New European guidelines represent a comprehensive, multidisciplinary approach to pulmonary embolism http://ow.ly/CFai2

Venous thromboembolism (VTE), which encompasses deep vein thrombosis and its most dangerous complication, acute pulmonary embolism (PE), represents a major threat for the health, the well-being and, under certain circumstances, the life of a large number of patients worldwide. With its acute and long-term complications, VTE also poses a substantial economic burden on national health systems. Alarmingly, since the risk of VTE approximately doubles with each decade after 40 years of age, it is to be expected that an ever larger number of individuals in "industrialised", ageing societies will be diagnosed with the disease in the years to come. In 2014, the results of landmark clinical trials and cohort studies, particularly those published in the preceding 6 years, were included in the update of the European Society of Cardiology (ESC) guidelines on the management of PE [1]. In the 2014 ESC guidelines, which were endorsed by the European Respiratory Society (ERS), several recommendations regarding diagnostic strategies and algorithms were retained and their validity reinforced and extended. In parallel, new data extending our knowledge with regards to optimal risk assessment and risk-adapted patient treatment led to major revisions in the recommendations in these latter areas, and new sections on the long-term sequelae of $\mathrm{PE}$ as well as its management in specific patient populations were added. The present article summarises and highlights the most relevant new aspects of the 2014 version as compared with the previous European guidelines published in 2008.

\section{Simplification of clinical prediction rules and age-adjusted D-dimer cut-offs}

Despite the limited sensitivity and specificity of individual symptoms, signs and common "baseline" clinical tests, the combination of findings evaluated by clinical judgement or by the use of standardised prediction rules allows us to classify patients with suspected PE into distinct categories of clinical, or pre-test, probability of the disease. Recently, both the Wells and the revised Geneva clinical prediction rule were simplified in order to increase their practicability and adoption into clinical practice $[2,3]$, and the simplified versions were externally validated $[4,5]$. Whichever rule or version is used, the proportion of patients with confirmed PE can be expected to be approximately $10 \%$ in the low-probability category, $30 \%$ in the intermediate-probability category and $65 \%$ in the high-clinical probability category when using the

Received: Sept 302014 | Accepted: Oct 022014

Support statement: The work of S. Konstantinides was supported by the German Federal Ministry of Education and Research (BMBF 01EO1003). The authors are responsible for the contents of this publication.

Conflict of interest: Disclosures can be found alongside the online version of this article at erj.ersjournals.com

Copyright @ERS 2014 
three-level classification [6]. If a two-level classification is used, the proportion of patients with confirmed $\mathrm{PE}$ in the PE-unlikely category is around $12 \%[6]$.

It has long been acknowledged that the specificity of a positive D-dimer test in suspected PE decreases steadily with age, to almost $10 \%$ in patients $>80$ years [7]. Using age-adjusted (instead of the conventional) cut-offs may improve the performance of D-dimer testing in the elderly. In a recent meta-analysis, ageadjusted cut-off values (age $\times 10 \mu \mathrm{g} \cdot \mathrm{L}^{-1}$, for age above 50 years) resulted in increased specificity in all age categories and particularly in patients aged $>80$ years, in whom specificity was more than doubled; sensitivity remained above $97 \%$ in all age groups [8]. A multicentre, prospective management study evaluated this age-adjusted cut-off level in a cohort of 3346 patients. Patients with a normal age-adjusted D-dimer value did not undergo computed tomographic (CT) pulmonary angiography; these patients were left untreated and followed over a 3-month period. On the basis of D-dimer, using the age-adjusted cut-off (instead of the "standard" $500 \mu \mathrm{g} \cdot \mathrm{L}^{-1}$ cut-off) increased the number of patients in whom PE could be excluded from $43(6.4 \%$; 95\% CI 4.8-8.5\%) to 200 (29.7\%; 95\% CI 26.4-33.3\%), without any additional false-negative findings [9].

\section{Persisting uncertainty in subsegmental and incidental PE}

The clinical significance of isolated subsegmental PE on CT pulmonary angiography is controversial. This finding was present in $4.7 \%(2.5-7.6 \%)$ of patients with PE imaged by single-detector CT angiography and $9.4 \%(5.5-14.2 \%)$ of those submitted to multidetector CT [10]. It is likely, although not yet confirmed by solid evidence, that a single subsegmental defect does not have the same clinical relevance as multiple, subsegmental thrombi. The positive predictive value is low and interobserver agreement is poor at this distal level [11]. Compression ultrasound of the leg veins may be helpful in this situation, as the exclusion of proximal deep vein thrombosis in a patient with isolated subsegmental PE would support a decision against anticoagulation treatment; such cases should be managed on an individual basis, taking into account the clinical probability and the bleeding risk.

The incidental discovery of clinically unsuspected PE on CT is an increasingly frequent problem, arising in $1-2 \%$ of all thoracic CT examinations, most often in patients with cancer, but also among those with paroxysmal atrial fibrillation, or heart failure and a history of atrial fibrillation [12-14]. There are no robust data to guide the decision on how to manage unsuspected PE, but most experts agree that patients with cancer and those with clots at the lobar or more proximal level should be treated with anticoagulants.

Advanced risk stratification of PE presenting without shock or persistent hypotension For prediction of early (in-hospital or 30 day) outcome in patients with acute PE, both the PE-related risk and the patient's clinical status and comorbidities should be taken into consideration. Generally, patients presenting without shock or hypotension, who represent the vast majority of patients with acute PE, are not at high risk of an adverse early outcome. However, further risk stratification should be considered in these patients after the diagnosis of $\mathrm{PE}$ has been confirmed, as this may influence the therapeutic strategy and the duration of hospitalisation. The 2014 PE guidelines recommend beginning "advanced" risk assessment of normotensive patients with confirmed PE with a validated clinical prognostic score, preferably the Pulmonary Embolism Severity Index (PESI) [15] or its simplified version (sPESI) [16], to distinguish between intermediate and low risk (of an adverse early outcome). This is in contrast to the previous (2008) guidelines, in which neither the PESI nor any other form of clinical assessment was an integral part of a structured risk stratification algorithm. Around one-third of PE patients are at low risk of an early adverse outcome as indicated by PESI class I or II, or an sPESI of 0 . By contrast, patients in PESI classes III or V have a 30 -day mortality rate of up to $24.5 \%$ [15], and those with a sPESI $\geqslant 1$ have a 30 -day mortality rate of up to $11 \%$ [16]. Accordingly, normotensive patients in PESI class $\geqslant I I I$, or an sPESI of $\geqslant 1$, constitute an intermediate-risk group. Within this category, further risk assessment should be considered, placing the focus on the status of the right ventricle (RV) in response to the PE-induced acute pressure overload. Patients who display evidence of both RV dysfunction (by echocardiography or CT angiography) and elevated cardiac biomarker levels in the circulation (particularly a positive cardiac troponin test) should be classified into an intermediate-high risk category. Patients in whom the RV is normal on echocardiography or CT angiography, and/or who have normal cardiac biomarker levels, belong to an intermediate-low risk group. The therapeutic implications of this classification are displayed in figure 1 and explained in the following sections.

Treatment and secondary prophylaxis of VTE with the new direct oral anticoagulants In patients with acute PE, anticoagulation effectively prevents both early death and recurrent symptomatic or fatal VTE. The duration of anticoagulation should cover at least 3 months. Within this period, the "standard" regimen of acute-phase treatment consists of administering parenteral anticoagulation 


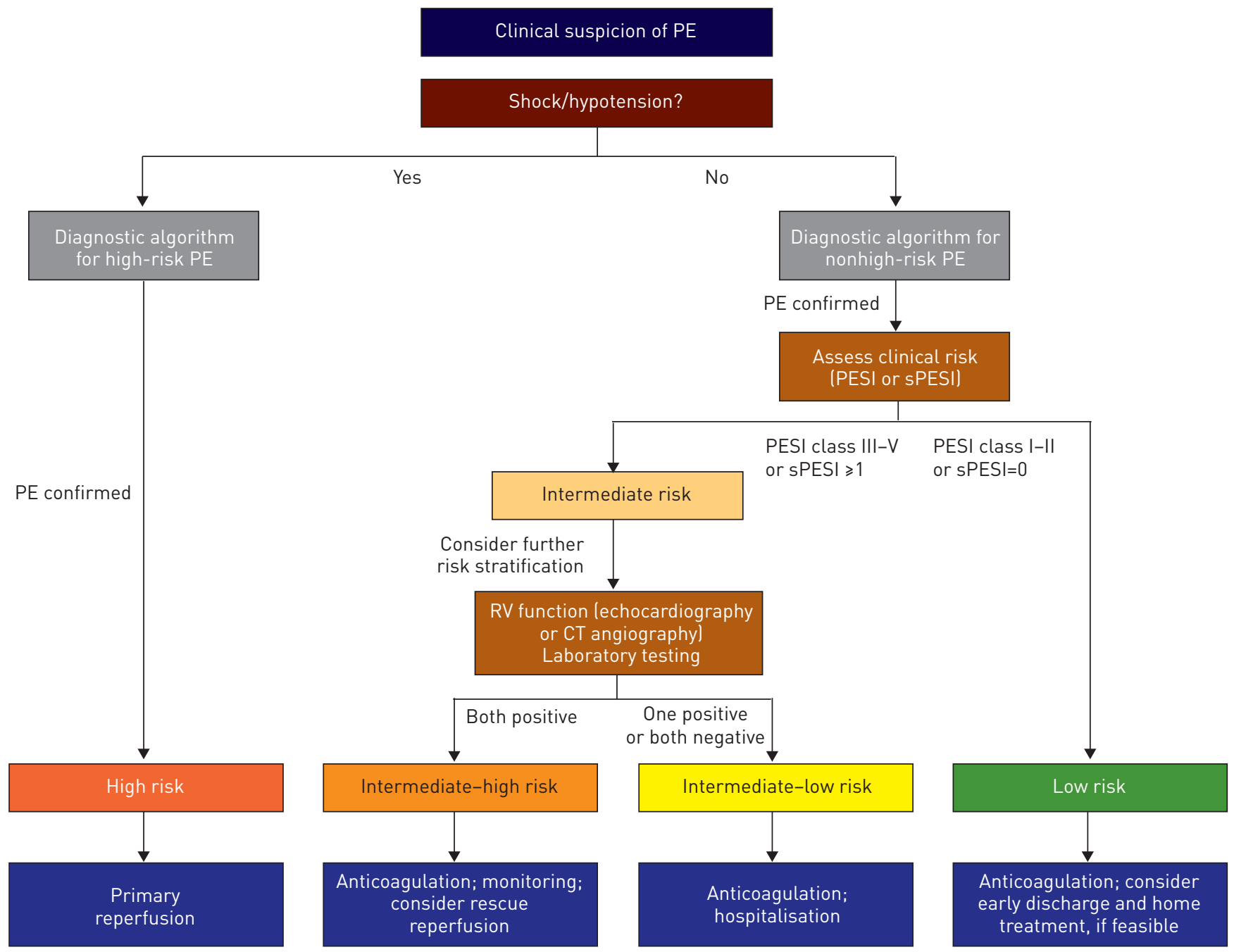

FIGURE 1 Risk-adjusted management strategies in acute pulmonary embolism (PE). PESI: pulmonary embolism severity index; sPESI: simplified PESI; RV: right ventricle; CT: computed tomography. Reproduced from [1] with permission from the publisher.

(unfractionated heparin, low molecular weight heparin or fondaparinux) over the first 5-10 days. Parenteral heparin should overlap with the initiation of a vitamin $\mathrm{K}$ antagonist. In some cases, extended anticoagulation beyond the first 3 months, or even indefinitely, may be necessary for secondary prevention, after weighing the individual patient's risk of recurrence versus bleeding risk.

The results of the trials using the new oral anticoagulants (NOACs) dabigatran [17, 18], rivaroxaban [19, 20], apixaban [21] or edoxaban [22] in the treatment of VTE indicated, both individually and in a metaanalysis [23], that these agents are non-inferior (in terms of efficacy) and possibly safer (particularly in terms of major bleeding) than the standard heparin/vitamin $\mathrm{K}$ antagonist regimen. As a result, NOACs are recommended in the $2014 \mathrm{PE}$ guidelines as an alternative to standard treatment. Currently, rivaroxaban, dabigatran and apixaban are approved for treatment of VTE in the European Union; edoxaban is under regulatory review. Experience with NOACs in clinical practice is still limited in VTE but has begun to accumulate at an increasing pace.

Three NOACs have also been evaluated in the extended treatment of patients with VTE: dabigatran [24], rivaroxaban [20] and apixaban [25]. To be included in the extended studies, patients needed to have completed the initial and long-term anticoagulation phase. In summary, the results of the trials using NOACs in the extended treatment of VTE are in line with those of the studies that tested these agents in the acute-phase treatment and standard duration of anticoagulation after PE or VTE. They indicate that NOACs are both effective (in terms of prevention of symptomatic or fatal recurrence of VTE) and safe (particularly in terms of major bleeding), and are probably safer than standard vitamin $\mathrm{K}$ antagonist 
regimens. As a result, the $2014 \mathrm{PE}$ guidelines state that treatment with a NOAC should be considered as an alternative to the use of a vitamin $\mathrm{K}$ antagonist if extended secondary prophylaxis is deemed necessary.

\section{Efficacy and safety of reperfusion treatment for patients at intermediate risk}

As mentioned above, an echocardiogram or CT scan indicating RV dysfunction in combination with a positive cardiac troponin test classifies a normotensive patient with PE into an intermediate-high risk group. Full-dose systemic thrombolytic therapy, given as primary reperfusion therapy, was shown to prevent potentially life-threatening haemodynamic decompensation or collapse in these patients, but this benefit was counterbalanced by a high risk of haemorrhagic stroke or major non-intracranial bleeding [26]. Accordingly, systemic thrombolysis is not routinely recommended as primary treatment for patients with intermediate-high risk PE, but should be considered if clinical signs of haemodynamic decompensation appear. Surgical pulmonary embolectomy or percutaneous catheter-directed treatment may be considered as alternative, "rescue" procedures for patients with intermediate-high risk PE, in whom haemodynamic decompensation appears imminent and the anticipated bleeding risk under systemic thrombolysis is high [27].

\section{Early discharge and home (outpatient) treatment of PE}

Recent multicentre clinical trials investigated the 3-month clinical outcome of patients with PE who were discharged early or treated entirely as outpatients. Overall, the proportion of screened patients who were identified as eligible for home treatment ranged from $13 \%$ to $51 \%$ [28]. In a meta-analysis of 14 (mostly cohort) studies, the pooled incidences of recurrent VTE, major bleeding and total mortality did not differ significantly between outpatients, patients discharged early, and those treated as inpatients [29]. Based on the evidence currently available and (partly) on expert opinion, low-risk patients in PESI class I or II, and probably those with an SPESI of 0 , should be considered for early discharge and outpatient treatment, if this appears feasible based on the patient's anticipated compliance as well as his/her family and social background. Further management trials are necessary to "crystallise" the criteria that might permit early discharge and home treatment of low-risk patients with acute PE.

\section{A new management algorithm for PE}

A comprehensive risk-adapted management algorithm for acute PE, which was developed and proposed for the first time in the $2014 \mathrm{PE}$ guidelines, is shown in figure 1.

Current diagnosis and treatment of chronic thromboembolic pulmonary hypertension Chronic thromboembolic pulmonary hypertension (CTEPH) is a debilitating disease caused by chronic obstruction of major pulmonary arteries. CTEPH has been reported to be a long-term complication of PE, with a reported cumulative incidence of $0.1-9.1 \%$ within the first 2 years after a symptomatic PE event [30]. This large margin of error is probably due to referral bias, absence of early symptoms, and the occasional difficulty in differentiating acute PE from an acute episode superimposed on pre-existing CTEPH.

The 2014 PE guidelines specify that CTEPH should be ruled out in PE survivors with persistent dyspnoea, but routine screening for CTEPH in asymptomatic survivors of PE is currently not recommended. In all patients with diagnosed CTEPH, the assessment of operability and decisions regarding other treatment strategies should be made by a multidisciplinary team of experts. Life-long anticoagulation is recommended in all patients with CTEPH, and surgical pulmonary endarterectomy (PEA) should be performed in those patients who are considered operable by a CTEPH team including at least one experienced PEA surgeon. Medical treatment with the recently approved agent riociguat is recommended in symptomatic patients who have been classified as having persistent/recurrent CTEPH after surgical treatment, or those with inoperable disease as judged by an expert CTEPH team (see earlier). An emerging interventional treatment option for "inoperable" patients, percutaneous balloon pulmonary angioplasty, is also mentioned in the updated CTEPH management algorithm.

\section{PE in specific patient populations}

The 2014 PE guidelines clearly state that suspicion of PE in pregnancy warrants formal diagnostic assessment with validated methods. Venous compression ultrasonography may be considered in order to avoid unnecessary irradiation, as a diagnosis of proximal deep vein thrombosis confirms PE. Perfusion scintigraphy may be deemed to rule out suspected PE in pregnant women with normal chest radiography. A weight-adjusted dose of low molecular weight heparin is the recommended therapy during pregnancy in patients without shock or hypotension.

The overall risk of VTE in cancer patients is four times as great as in the general population [31]. Although the largest absolute numbers of VTE episodes occur in patients with lung, colon and prostate cancer, the relative risk for VTE is highest in multiple myeloma, brain, and pancreatic cancer (46-, 20-, and 16-fold 
increased relative risk versus healthy controls, respectively) [32]. Patients receiving chemotherapy have a sixfold increase in the adjusted risk ratio for VTE compared with a healthy population [31]. Nevertheless, prophylactic anticoagulation is not routinely recommended during ambulatory anticancer chemotherapy, with the exception of thalidomide- or lenalidomide-based regimens in multiple myeloma [33, 34]. The risk of VTE increases over 90-fold in the first 6 weeks after cancer surgery, compared with that in healthy controls, and is second only to the risk of VTE after hip or knee replacement surgery. For patients with PE and cancer, weight-adjusted subcutaneous low molecular weight heparin should be considered for the first 3-6 months; evidence with new oral anticoagulants is still limited in this setting. For patients with PE and cancer extended anticoagulation (beyond the first 3-6 months) should be considered for an indefinite period, or until the cancer is cured.

\section{Conclusions}

The medical community is finally becoming aware of the epidemiological and clinical relevance of VTE, the third most frequent acute cardiovascular syndrome after myocardial infarction and stroke. Considerable progress in the diagnosis, treatment and prevention of the disease has been made in the past few years, and management concepts continue to evolve at a rapid pace. Based on the recent advances in our knowledge and skills, and thanks to the joint efforts of experts nominated by the ESC and the ERS in close collaboration, the new European PE guidelines represent a contemporary, truly multidisciplinary approach to PE and VTE. The guidelines Task Force took every care to be as thorough, as impartial, and as evidencebased as possible in its recommendations, while at the same time providing a practical, user-friendly guide to physicians involved in the management of PE in Europe and worldwide.

\section{References}

1 Konstantinides SV, Torbicki A, Agnelli G, et al. 2014 ESC Guidelines on the diagnosis and management of acute pulmonary embolism: the Task Force for the Diagnosis and Management of Acute Pulmonary Embolism of the European Society of Cardiology (ESC). Endorsed by the European Respiratory Society (ERS). Eur Heart J 2014 [In press DOI: 10.1093/eurheartj/ehu283].

2 Gibson NS, Sohne M, Kruip MJ, et al. Further validation and simplification of the Wells clinical decision rule in pulmonary embolism. Thromb Haemost 2008; 99: 229-234.

3 Klok FA, Mos IC, Nijkeuter M, et al. Simplification of the revised Geneva score for assessing clinical probability of pulmonary embolism. Arch Intern Med 2008; 168: 2131-2136.

4 Douma RA, Mos IC, Erkens PM, et al. Performance of 4 clinical decision rules in the diagnostic management of acute pulmonary embolism: a prospective cohort study. Ann Intern Med 2011; 154: 709-718.

5 Douma RA, Gibson NS, Gerdes VE, et al. Validity and clinical utility of the simplified Wells rule for assessing clinical probability for the exclusion of pulmonary embolism. Thromb Haemost 2009; 101: 197-200.

6 Ceriani E, Combescure C, Le Gal G, et al. Clinical prediction rules for pulmonary embolism: a systematic review and meta-analysis. J Thromb Haemost 2010; 8: 957-970.

7 Righini M, Goehring C, Bounameaux $\mathrm{H}$, et al. Effects of age on the performance of common diagnostic tests for pulmonary embolism. Am J Med 2000; 109: 357-361.

8 Schouten HJ, Geersing GJ, Koek HL, et al. Diagnostic accuracy of conventional or age adjusted D-dimer cut-off values in older patients with suspected venous thromboembolism: systematic review and meta-analysis. BMJ 2013; 346: f2492.

9 Righini M, Van Es J, den Exter PL, et al. Age-adjusted D-dimer cutoff levels to rule out pulmonary embolism: the ADJUST-PE study. JAMA 2014; 311: 1117-1124.

10 Carrier M, Righini M, Wells PS, et al. Subsegmental pulmonary embolism diagnosed by computed tomography: incidence and clinical implications. A systematic review and meta-analysis of the management outcome studies. J Thromb Haemost 2010; 8: 1716-1722.

11 Stein PD, Goodman LR, Hull RD, et al. Diagnosis and management of isolated subsegmental pulmonary embolism: review and assessment of the options. Clin Appl Thromb Hemost 2012; 18: 20-26.

12 Jia CF, Li YX, Yang ZQ, et al. Prospective evaluation of unsuspected pulmonary embolism on coronary computed tomographic angiography. J Comput Assist Tomogr 2012; 36: 187-190.

13 Palla A, Rossi G, Falaschi F, et al. Is incidentally detected pulmonary embolism in cancer patients less severe? A case-control study. Cancer Invest 2012; 30: 131-134.

14 Sahut D'Izarn M, Caumont Prim A, Planquette B, et al. Risk factors and clinical outcome of unsuspected pulmonary embolism in cancer patients: a case-control study. J Thromb Haemost 2012; 10: 2032-2038.

15 Aujesky D, Obrosky DS, Stone RA, et al. Derivation and validation of a prognostic model for pulmonary embolism. Am J Respir Crit Care Med 2005; 172: 1041-1046.

16 Jiménez D, Aujesky D, Moores L, et al. Simplification of the pulmonary embolism severity index for prognostication in patients with acute symptomatic pulmonary embolism. Arch Intern Med 2010; 170: 1383-1389.

17 Schulman S, Kakkar AK, Goldhaber SZ, et al. Treatment of acute venous thromboembolism with dabigatran or warfarin and pooled analysis. Circulation 2014; 129: 764-772.

18 Schulman S, Kearon C, Kakkar AK, et al. Dabigatran versus warfarin in the treatment of acute venous thromboembolism. N Engl J Med 2009; 361: 2342-2352.

19 Büller HR, Prins MH, Lensin AW, et al. Oral rivaroxaban for the treatment of symptomatic pulmonary embolism. N Engl J Med 2012; 366: 1287-1297.

20 Bauersachs R, Berkowitz SD, Brenner B, et al. Oral rivaroxaban for symptomatic venous thromboembolism. $N$ Engl J Med 2010; 363: 2499-2510. 
21 Agnelli G, Buller HR, Cohen A, et al. Oral apixaban for the treatment of acute venous thromboembolism. $N$ Engl J Med 2013; 369: 799-808.

22 Büller HR, Décousus H, Grosso MA, et al. Edoxaban versus warfarin for the treatment of symptomatic venous thromboembolism. N Engl J Med 2013; 369: 1406-1415.

23 van der Hulle T, Kooiman J, den Exter PL, et al. Effectiveness and safety of novel oral anticoagulants as compared with vitamin $\mathrm{K}$ antagonists in the treatment of acute symptomatic venous thromboembolism: a systematic review and meta-analysis. J Thromb Haemost 2014; 12: 320-328.

24 Schulman S, Kearon C, Kakkar AK, et al. Extended use of dabigatran, warfarin, or placebo in venous thromboembolism. N Engl J Med 2013; 368: 709-718.

25 Agnelli G, Buller HR, Cohen A, et al. Apixaban for extended treatment of venous thromboembolism. N Engl J Med 2013; 368: 699-708.

26 Meyer G, Vicaut E, Danays T, et al. Fibrinolysis for patients with intermediate-risk pulmonary embolism. $N$ Engl J Med 2014; 370: 1402-1411.

27 Kucher N, Boekstegers P, Müller OJ, et al. Randomized, controlled trial of ultrasound-assisted catheter-directed thrombolysis for acute intermediate-risk pulmonary embolism. Circulation 2014; 129: 479-486.

28 Lankeit M, Konstantinides S. Is it time for home treatment of pulmonary embolism? Eur Respir J 2012; 40: 742-749.

29 Zondag W, Kooiman J, Klok FA, et al. Outpatient versus inpatient treatment in patients with pulmonary embolism: a meta-analysis. Eur Respir J 2013; 42: 134-144.

30 Lang IM, Pesavento R, Bonderman D, et al. Risk factors and basic mechanisms of chronic thromboembolic pulmonary hypertension: a current understanding. Eur Respir J 2013; 41: 462-468.

31 Heit JA, Silverstein MD, Mohr DN, et al. Risk factors for deep vein thrombosis and pulmonary embolism: a population-based case-control study. Arch Intern Med 2000; 160: 809-815.

32 Cronin-Fenton DP, Søndergaard F, Pedersen LA, et al. Hospitalisation for venous thromboembolism in cancer patients and the general population: a population-based cohort study in Denmark, 1997-2006. Br J Cancer 2010; 103: 947-953.

33 Akl EA, Vasireddi SR, Gunukula S, et al. Anticoagulation for patients with cancer and central venous catheters. Cochrane Database Syst Rev 2011; 4: CD006468.

34 Palumbo A, Cavo M, Bringhen S, et al. Aspirin, warfarin, or enoxaparin thromboprophylaxis in patients with multiple myeloma treated with thalidomide: a phase III, open-label, randomized trial. J Clin Oncol 2011; 29: 986-993. 